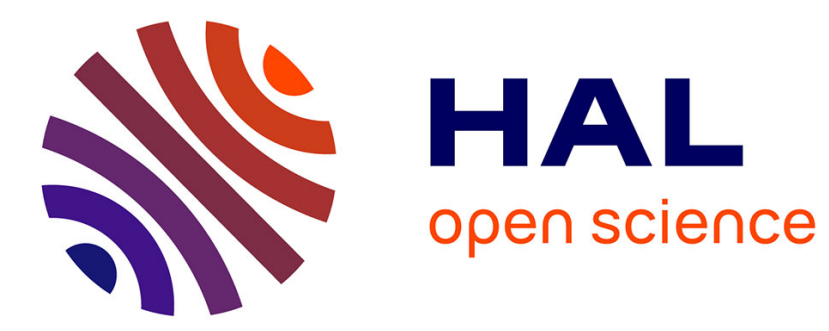

\title{
Increase in Multicast OFDM Data Rate in PLC Network using Adaptive LP-OFDM
}

Ali Maiga, Jean-Yves Baudais, Jean-François Hélard

\section{To cite this version:}

Ali Maiga, Jean-Yves Baudais, Jean-François Hélard. Increase in Multicast OFDM Data Rate in PLC Network using Adaptive LP-OFDM. IEEE ICAST, Dec 2009, Accra, Ghana. pp.384 - 389. hal-00444406

\section{HAL Id: hal-00444406 https://hal.science/hal-00444406}

Submitted on 6 Jan 2010

HAL is a multi-disciplinary open access archive for the deposit and dissemination of scientific research documents, whether they are published or not. The documents may come from teaching and research institutions in France or abroad, or from public or private research centers.
L'archive ouverte pluridisciplinaire HAL, est destinée au dépôt et à la diffusion de documents scientifiques de niveau recherche, publiés ou non, émanant des établissements d'enseignement et de recherche français ou étrangers, des laboratoires publics ou privés. 


\title{
Increase in Multicast OFDM Data Rate in PLC Network using Adaptive LP-OFDM
}

\author{
Ali Maiga, Jean-Yves Baudais and Jean-François Hélard \\ Institute of Electronics and Telecommunications of Rennes \\ 35043 Rennes Cedex, France \\ Email: \{ali.maiga, jean-yves.baudais, jean-francois.helard\}@insa-rennes.fr
}

\begin{abstract}
Linear precoding (LP) technique applied to OFDM systems has already proved its ability to significantly increase the system throughput in a powerline communication (PLC) context. In this paper, we propose resource allocation algorithms based on the LP technique to increase the multicast OFDM systems bit rate. The conventional multicast capacity is limited by the user which experiences the worst channel conditions. To increase the multicast bit rate, these proposed algorithms assign subcarriers and bits to different multicast users. Simulations are run over PLC channels and it is shown that the proposed solutions offer a bit rate gain up to $37 \%$ compared to the conventional multicast bit rate.
\end{abstract}

\section{INTRODUCTION}

Initially developed in the 1980's for low bit rate applications, such as telemetry, infrastructure control and consumption measurement, powerline communication (PLC) technologies have recently drawn an increasing interest within the scientific community for high bit rate communications over the power grid. Due to significant advances achieved in terms of modulation and signal processing schemes on one hand, and owing to the ubiquity of the outlet on the other hand, PLC is today considered as a solution of high potential for indoor applications and constitutes a convenient and cheap alternative to already existing technologies.

We are witnessing the increase of data applications with high bandwidth requirements and the demand for multimedia services such as video and audio conferencing, online training is increasing. So far, the multimedia services are unicasted (brought separately) to each user of the PLC network, leading to data duplication and lost of time and bandwidth. For these applications, multicasting offers a significant improvement compared to unicasting, since it allows the transmission of packets to multiple destinations using less resources [1]. The powerline channel exhibits multipaths caused by reflections on the discontinuities of the network and offers impulse responses that can be assumed as quasi-static and encourages the exploitation of the channel state information (CSI) at the transmitter side. Yet, the difference in link conditions of users makes it difficult to apply resource allocation process because the requirements of each multicast user should be satisfied. The conventional resource allocation method in multicast orthogonal frequency division multiplexing (OFDM) is to serve the "worst" user. Consequently, this method may not provide efficient performances when the number of multicast users increases.
To increase the multicast bit rate, it has been suggested to exploit hierarchy in multicast data, when employing multiresolution coding [1-3]. The data is compressed into a number of layers, arranged in a hierarchy, which provides progressive refinement. If only the first layer is received by the user with the lowest data rate, the decoder produces the worst quality version. As more layers are received by more capable users, the decoder combines the layers to produce improved quality [1]. Few studies have been carried out on multicast OFDM systems for PLC, and even lesser studies seeking to increase the multicast bit rate without using hierarchical data.

In this paper, resource allocation algorithms for multicast OFDM systems are proposed in order to increase the data rate in non-hierarchical data context. These algorithms allocate dynamically subcarriers and bits and are based on the linear precoding technique. Due to power spectral density (PSD) constraint in PLC systems, all users have the same peak power constraint on each subcarrier and there is no power allocation. Linear precoded OFDM (LP-OFDM) is a combination of multicarrier and spread spectrum techniques also known as MC-SS techniques. This technique has shown very good performances in difficult environments and brings a significant increase in bit rate compared to classical OFDM systems [4], [5], [6]. In the following, it is discussed how to take advantage of linear precoding technique for multicast services in multicarrier systems. New resource allocation algorithms are proposed for LP-OFDM to increase the multicast bit rate.

This paper is organized as follows. Section II describes the LP-OFDM systems. Section III presents the optimization problem in multicast OFDM systems. Section IV gives the proposed solutions to the optimization problem. The performances of these solutions are given in section $\mathrm{V}$ over PLC channels. Finally, section VI concludes the paper.

In the following, upper (resp. lower) boldface symbols are used for matrices (resp. column vector), lower symbols represent scalar values. Besides, $(.)^{T}$ and $(.)^{H}$ denote transpose and Hermitian operators respectively, and $I_{N}$ stands for the $N \times N$ identity matrix.

\section{LP-OFDM SYSTEM DESCRIPTION}

\section{A. Adaptive linear precoded OFDM system model}

The studied LP-OFDM system is based on the combination of OFDM with linear precoding. In a general approach, the 
$N$-dimensional generated symbol vector $\mathbf{s}$ can be written as

$$
\mathbf{s}=\mathbf{F}^{H} \mathbf{W} \mathbf{x} .
$$

Vector $\mathbf{x}=\left[x_{1}, \ldots, x_{K}\right]^{T}$ is the result of the serial to parallel conversion of the input data stream containing the $K$ QAM-mapped symbols to transmit. W stands for the $N \times K$ precoding matrix applied to $\mathbf{x}$ which precodes $K$ symbols over $N$ subcarriers. The precoded symbol vector is finally multiplied by the $N \times N$ unitary Fourier matrix $\mathbf{F}$ that realizes the multicarrier modulation. $N$ represents the number of subcarriers used in the system. Note that for $\mathbf{W}=\mathbf{I}_{N}$, the system amounts to the classical non-precoded OFDM system.

Depending on the structure of the precoding matrix, it is in fact possible to precode the symbol stream following different strategies. In this paper, we focus on a particular instance of this broad category of LP-OFDM signals where the precoding function is applied to different blocks of subcarriers independently. The block model of the proposed LP-OFDM system is depicted in Fig. 1 and the resulting signal is then

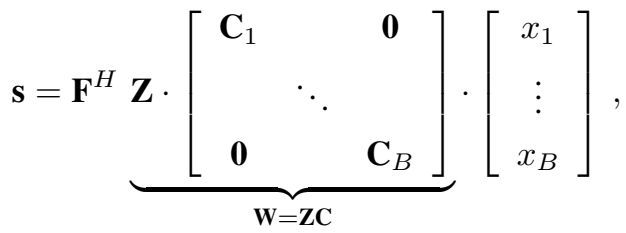

with $B$ the number of blocks to consider. Matrices $\mathbf{C}_{b}=$ $\left\{c_{l, k}\right\}_{1 \leq l \leq L_{b}, 1 \leq k \leq K_{b}}$ are the individual precoding matrices containing the $K_{b}$ precoding sequences of length $L_{b}$ to apply to each block $S_{b}, b=[1 ; B]$. Note that $L_{b}$ is also equivalent to the size of the $b$ th block $S_{b}$, i.e. to the number of gathered subcarriers in that block. From (1), we can write $\sum_{b} L_{b}=N$, and $\sum_{b} K_{b}=K . \mathbf{x}_{b}=\left[x_{b, 1}, \ldots, x_{b, K_{b}}\right]^{T}$ are $K_{b}$-dimensional vectors of QAM symbols to be transmitted within the different blocks. We use M-QAM constellations of orders $m_{b, k} \in[1 ; 10]$ like in PLC systems. Within each block, note that each QAM symbol $x_{b, k}$ is associated to a certain precoding sequence. Finally, $\mathbf{Z}$ is a permutation matrix that basically realizes an interleaving of the precoded QAM symbols in the frequency domain. Consequently, one has to keep in mind that blocks are not necessarily made of adjacent subcarriers.

After classical cyclic prefix extension, the generated data stream is sent across the PLC channel. Assuming perfect synchronization and cyclic prefix removal at the receiver, the channel can be modeled by one single complex coefficient per subcarrier [5]. After multicarrier demodulation the received signal is then

$$
\mathbf{y}=\mathbf{H W x}+\mathbf{n}_{0},
$$

where $\mathbf{H}=\operatorname{diag}\left(\left[h_{1}, \ldots, h_{N}\right]\right)$ is the $N \times N$ diagonal complex matrix bearing on its diagonal the frequency domain channel gains associated to each subcarrier $n \in[1 ; N]$. Vector $\mathbf{n}_{0}$ represents the additive white Gaussian noise such that $\mathrm{E}\left[\mathbf{n}_{0} \mathbf{n}_{0}^{H}\right]=N_{0} \mathbf{I}_{N}$. The channel effects are finally compensated for using a simple zero-forcing (ZF) equalizer before desinterleaving and inverse precoding operations. Denoting $\mathbf{G}=\mathbf{H}^{-1}$

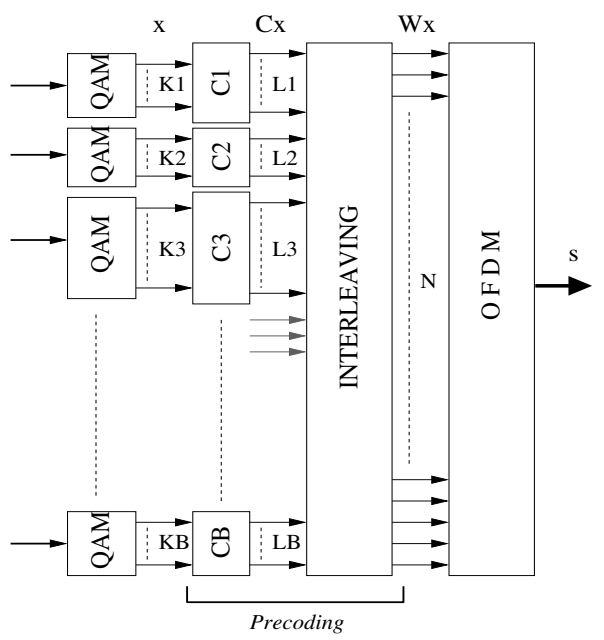

Fig. 1. Proposed LP-OFDM transmitter

the diagonal equalization matrix, the received vector of data symbol d then writes

$$
\mathbf{d}=\mathbf{W}^{-1} \mathbf{G y}
$$

The following expressions are derived from [5], [6], where there are more details. The optimum achieved bit rate of the LP-OFDM system using ZF detection is given by

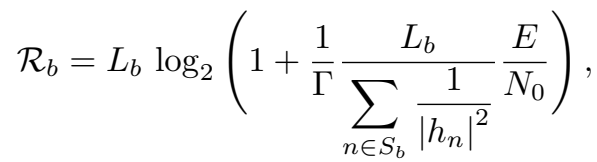

where $E$ is the PSD constraint, $\Gamma$ is the signal-to-noise ratio (SNR) gap and $K_{b}=L_{b}$. The classical OFDM system is obtained for $L_{b}=1$.

\section{Choice of the $L_{b}$ subcarriers}

To maximize the bit rate $\mathcal{R}_{b}$ given by (5), it suffices to minimize the sum $\sum_{n \in S_{b}} \frac{1}{\left|h_{n}\right|^{2}}$. This corresponds to choose the subcarriers with best channel gains $\left|h_{n}\right|^{2}$. A simpler solution is to sort subcarriers in descending order and to choose the first $L_{b}$ subcarriers.

\section{B. Performance of LP-OFDM in PLC context}

In our study, the LP component is used to multiplex different data symbols of a given user. The multiplexed data symbols are sent in subsets of subcarriers which are merged with precoding sequences. If the merging process is judiciously done, each resulting subset holds an equivalent SNR such that the total supported throughput is greater than the sum of the individual throughputs supported by each subcarrier taken separately. Fig. 2 shows the performance of LP-OFDM system over a Rayleigh fading channel in single user context. The precoding sequence length is equal to 4 or 32. Fig. 2a shows the loaded bit per subcarrier for OFDM and LP-OFDM bitloading algorithms when the average channel gain varies. The LP-OFDM system increases the classical OFDM system bit 
(a)

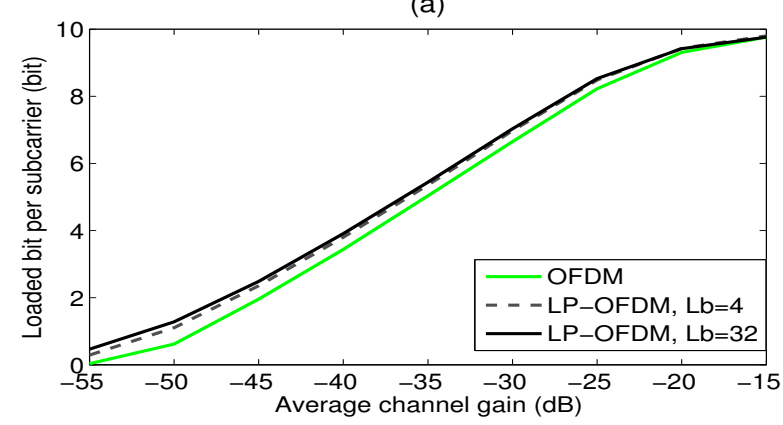

(b)

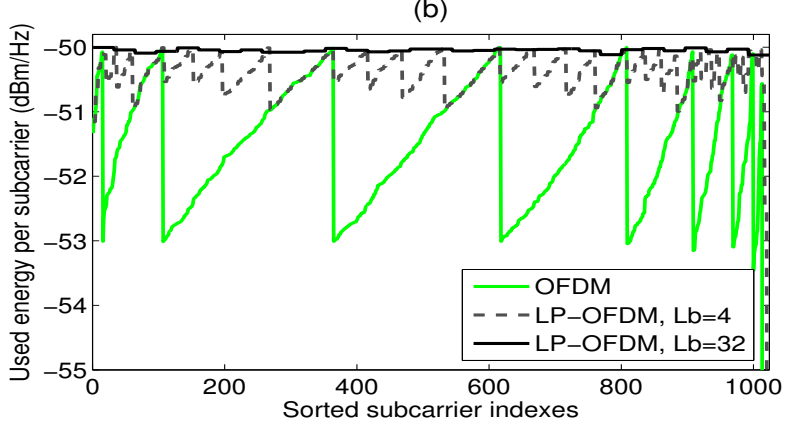

Fig. 2. Performance of LP-OFDM system over a Rayleigh fading channel in single user context. (a) loaded bit per subcarrier; (b) used energy per subcarrier for average channel gain of $-30 \mathrm{~dB}$.

rate and the gain brought by the linear precoding component is more important for a longer precoding sequence.

The reason for the better performance of the LP-OFDM system is explained in Fig. 2b, where the used energy of different algorithms are compared. To highlight the energy distribution, subcarriers are sorted in descending order. The used energy is the minimal required energy allowing the transmission of the maximum data rate. Jump positions in curves correspond to the change in modulation orders. It is clear that the OFDM algorithm is not fully exploiting the available energy on each subcarrier due to discrete modulation orders. The precoding component accumulates the residual lost energies of a given block of subcarriers to transmit additional bits. The adaptive LP-OFDM system utilizes more efficiently the PSD limit. The longer the precoding sequence, the better the used energy. However, a compromise on precoding sequence length has to be made because the self interference (interference between precoding sequences) increases with the precoding sequence length, due to channel selectivity.

The additional complexities of LP-OFDM are the precoding matrix which is composed of Hadamard orthogonal matrices, and the interleaver at the transmitter side. At the receiver side, their inverse functions are used.

\section{BIT RATE OPTIMIZATION PROBLEM IN MULTICAST OFDM SYSTEMS}

In multicast OFDM systems, the modulation should be adjusted to serve the user with the worst channel conditions, in non-hierarchical data context. The classical method in multicast OFDM, LCG (low channel gain, [1]) consists in allocating resources while satisfying requirements of all users. This method sets the achieved bit rate per subcarrier with the lowest bit rate of users over this subcarrier. Hence, the achieved bit rate with LCG method over subcarrier $n$ in PLC context writes

$$
\mathcal{R}_{n}^{\mathrm{LCG}}=\min _{u} \log _{2}\left(1+\frac{E}{\Gamma N_{0}}\left|h_{u, n}\right|^{2}\right),
$$

where $\left|h_{u, n}\right|^{2}$ is the channel gain of user $u$ on subcarrier $n$.

When considering the LP-OFDM system, the achieved multicast bit rate over the block $S_{b}$ of subcarriers will be the lowest bit rate of users over this block. This bit rate writes

$$
\mathcal{R}_{b}^{\mathrm{LP}}=\min _{u} \mathcal{R}_{u, b}=\min _{u} L_{b} \log _{2}\left(1+\frac{1}{\Gamma} \frac{L_{b}}{\sum_{n \in S_{b}} \frac{1}{\left|h_{u, n}\right|^{2}}} \frac{E}{N_{0}}\right) .
$$

Due to PSD constraint in PLC systems, all users have the same peak power constraint $E$ on each subcarrier. Hence, there is no power allocation. For simplicity, it is assumed that all users utilize the same precoding sequence length $L$ for all blocks. The block index $b$ can then be removed in the following for the precoding sequence length.

To maximize the multicast bit rate, we need to choose the block $S_{b}$ that maximizes the "worst" user bit rate. As,

$$
\begin{aligned}
& \min \mathcal{R}_{u, b} \Leftrightarrow \max \sum_{n \in S_{b}} \frac{1}{\left|h_{u, n}\right|^{2}} \\
& \text { and } \max \mathcal{R}_{u, b} \Leftrightarrow \min \sum_{n \in S_{b}} \frac{1}{\left|h_{u, n}\right|^{2}},
\end{aligned}
$$

the optimization problem writes

$$
\min _{S_{b}} \max _{u} \sum_{n \in S_{b}} \frac{1}{\left|h_{u, n}\right|^{2}} .
$$

This is a combinatorial and min-max-sum resource allocation problem which is an NP-hard problem [7].

\section{PROPOSED SOLUTIONS TO THE OPTIMIZATION PROBLEM}

In (8), we need to find the optimal $S_{b}$ which maximizes the "worst" user bit rate. Proposed solutions to distribute subcarriers in blocks are given in the following. A basic resolution which consists in testing all possibilities of defining blocks is first analyzed. Then, a two-step heuristic procedure is proposed. This procedure aims at finding the optimal block for each multicast user before selecting the best case. Finally, an equivalent channel which is the combination of users channel conditions is generated. This equivalent channel is used to define the blocks of subcarriers. 


\section{A. Combinatorial solution}

The basic resolution of finding the optimal block of subcarriers is to test all possibilities of gathering subcarriers in blocks and then to choose the best case. As both the order of subcarrier indexes within a block and the order of blocks do not change the result, the number of possibilities can be considered as

$$
\frac{C_{N}^{L} \times C_{N-L}^{L} \times C_{N-2 L}^{L} \times \ldots \times C_{2 L}^{L}}{\left(\frac{N}{L}\right) !}=\frac{N !}{(L !)^{\left(\frac{N}{L}\right)}\left(\frac{N}{L}\right) !},
$$

where the number $N$ of subcarriers is multiple of the number $L$ of subcarriers per block. This number of possibilities depicts the number of combinations of $L$ out of $N$, and the number of combinations of $L$ out of $N-L$, until all combinations are taken into account. Then, the total number of combinations is divided by the number of arrangements of blocks. For $N=$ 16 and $L=4$, the number of possibilities, needed to find the optimum multicast bit rate, is more than 10 million. This number is very high and will lead to high simulation time.

\section{B. Heuristic solution}

In this solution, each user is considered as the "worst" user and we try to choose the optimal block of subcarriers. The best bit rate of users defines the multicast bit rate. Considering the optimization problem (8), the following can be derived $\forall u$

$$
u^{*}=\arg \max _{u} \sum_{n \in S_{b}} \frac{1}{\left|h_{u, n}\right|^{2}} \Leftrightarrow \sum_{n \in S_{b}} \frac{1}{\left|h_{u^{*}, n}\right|^{2}} \geq \sum_{n \in S_{b}} \frac{1}{\left|h_{u, n}\right|^{2}} .
$$

Assuming that $u^{*}$ is known, the problem (8) can be converted into classical minimization problem under constraints

$$
\left\{\begin{array}{l}
\min _{S_{b}} \sum_{n \in S_{b}} \frac{1}{\left|h_{u^{*}, n}\right|^{2}} \\
\text { subject to } \sum_{n \in S_{b}} d_{u^{*}, v, n} \geq 0,
\end{array}\right.
$$

where,

$$
d_{u^{*}, v, n}=\frac{1}{\left|h_{u^{*}, n}\right|^{2}}-\frac{1}{\left|h_{v, n}\right|^{2}},
$$

where $v$ is the index of other users. Hence, a solution to problem (10) will consist in a two-step approach. Considering that the system has $B$ blocks of subcarriers, this method optimizes the multicast system bit rate for each block.

1) For each multicast user $u$, find the block $S_{b}^{u}$ of available subcarriers, where the user achieves its best minimum bit rate.

2) Choose the user who achieves the best minimum bit rate and its corresponding block $S_{b}^{u}$.

Considering a system with $U$ users, $N$ subcarriers that can be gathered in $B$ blocks of $L$ subcarriers, the algorithm 1 gives a solution of problem (10).

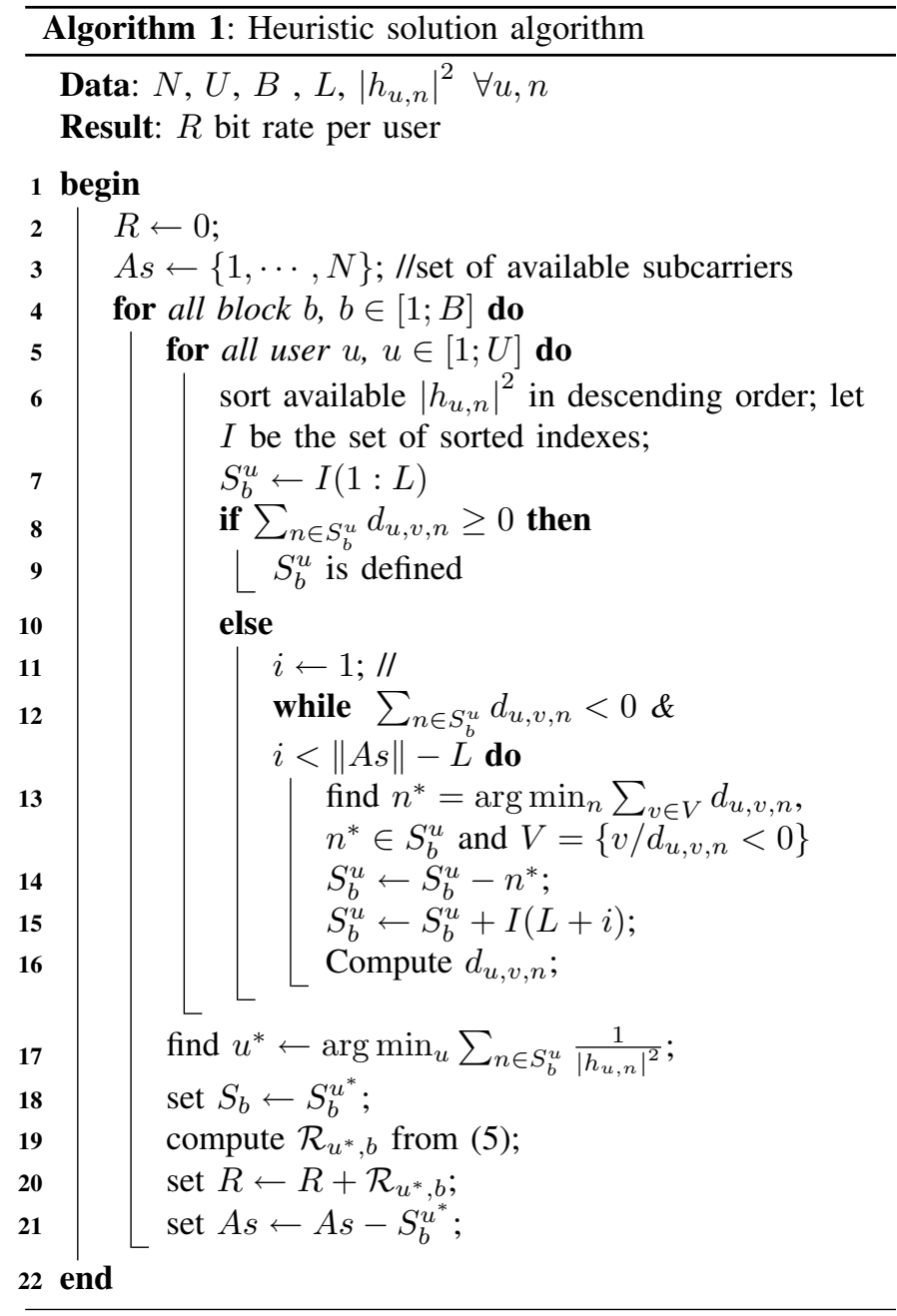

\section{Exploitation of classical LCG method}

In the LCG method, the multicast bit rate can be considered as the bit rate computed over an equivalent channel in single user context. This equivalent multicast channel is the combination of channel conditions of different users. Actually, for each index of subcarrier, the equivalent gain of the channel is given by the gain of the "worst" user subcarrier. Let $\left|h_{n}^{\mathrm{eq}}\right|^{2}$ be the equivalent multicast channel gain on subcarrier $n$. Hence,

$$
\left|h_{n}^{\mathrm{eq}}\right|^{2}=\min _{u}\left|h_{u, n}\right|^{2} .
$$

Computing this equivalent multicast channel, the multicast resource allocation is the same as the single link resource allocation. Bit-loading algorithms in single user context can then be applied to this channel. LCG method gives results for classical OFDM bit-loading algorithm. To increase the bit rate of this LCG method, the LP-OFDM bit-loading in single user context [4], is applied on the equivalent channel and this method will be considered as linear precoding based LCG (LPLCG) method. Then, subcarriers of the equivalent channel are sorted in descending order and best available subcarriers are gathered in a block. 


\section{Proposed Improved-LP-LCG method}

Considering blocks of subcarriers, we show, for all $n \in S_{b}$ and for all $u$,

$$
\begin{aligned}
\left|h_{u, n}\right|^{2} \geq\left|h_{n}^{\mathrm{eq}}\right|^{2} & \Leftrightarrow \max _{u} \sum_{n \in S_{b}} \frac{1}{\left|h_{u, n}\right|^{2}} \leq \sum_{n \in S_{b}} \frac{1}{\left|h_{n}^{\mathrm{eq}}\right|^{2}} \\
& \Leftrightarrow \min _{S_{b}} \max _{u} \sum_{n \in S_{b}} \frac{1}{\left|h_{u, n}\right|^{2}} \leq \min _{S_{b}} \sum_{n \in S_{b}} \frac{1}{\left|h_{n}^{\mathrm{eq}}\right|^{2}} .
\end{aligned}
$$

From (13), we derive that the "worst" user over block $S_{b}$ offers a better bit rate than the LP-LCG method. We aim at finding the solution of the left hand side of inequality (13) according to (8). Minimize the right hand side of the inequality reduces the left hand side and using the best available $L$ subcarriers of the equivalent channel to form $S_{b}$ minimizes the right hand side. This solution, called Improved-LP-LCG, consists in sorting the subcarriers of the equivalent channel in descending order and the indexes of sorted subcarriers define the different blocks. The algorithm 2 describes how to compute the multicast bit rate with the improved-LP-LCG method. Results for the classical LCG method is obtained for $L=1$.

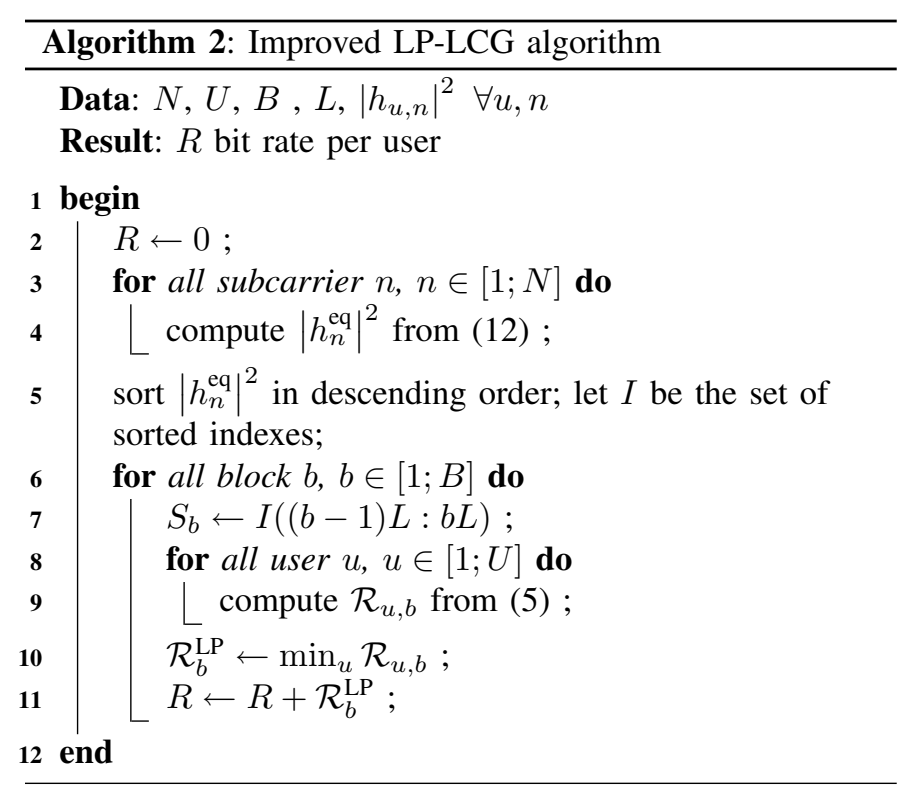

\section{E. Comparison of different solutions}

For a fix precoding sequence length, the combinatorial solution gives the optimum multicast bit rate, but the number of possibilities to be tested significantly increases when the number of subcarriers increases. Fig. 3 gives the comparison of proposed combinatorial solution and others, over an i.i.d. Rayleigh fading channel. The total number of subcarriers is $N=12$ and the precoding sequence length is $L=4$. As expected, the combinatorial solution outperforms the other ones and the LP component improves the classical multicast OFDM system (LCG). The improved-LP-LCG method gives performance near the combinatorial one when the number

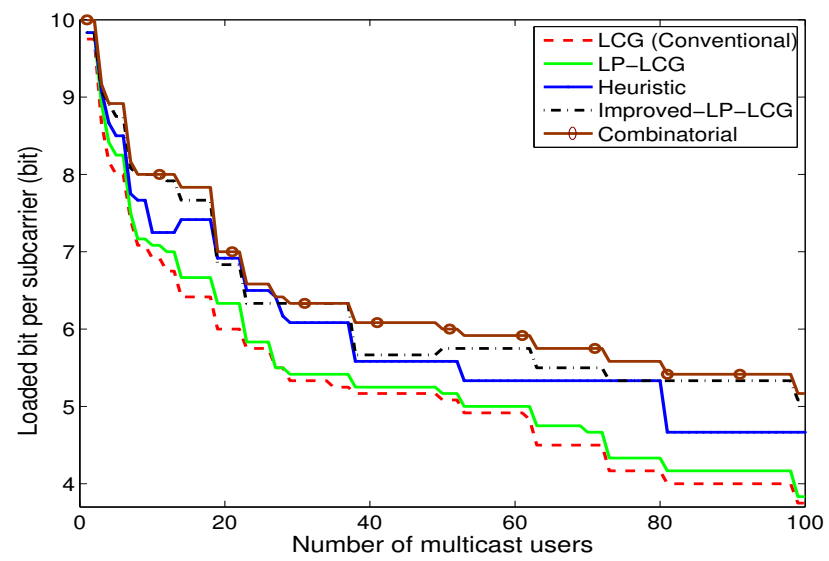

Fig. 3. Comparison of combinatorial solution with others for $N=12$ and $L=4$ over Rayleigh fading channels

of users increases. Yet, this tendency cannot be generalized to a given number $N$ because of difficulties in performing the combinatorial solution when $N$ is high. In the following, simulations are not performed for the combinatorial solution.

Compared to the classical LCG method, the LP-LCG method brings the same additional complexity as the LPOFDM system to classical OFDM system. The improvement of this LP-LCG method (Improved-LP-LCG) needs the computation of different $\mathcal{R}_{u, b}$. Table I shows the different computational times over Rayleigh fading channel with 12 subcarriers and 100 users.

\begin{tabular}{|c|c|c|c|c|}
\hline Algorithm & LCG & LP-LCG & Improved-LP-LCG & Heuristic \\
\hline \multirow{2}{*}{ Time (ms) } & 0.326 & 0.424 & 2.013 & 50.98 \\
& $(0.490)$ & $(0.785)$ & $(3.673)$ & $(53.44)$ \\
\hline
\end{tabular}

TABLE I

MEAN (WORST CASE) COMPUTATION TIMES IN MILLISECONDS FOR N $\mathrm{N} 12$, $\mathrm{U}=100$ AND L=4 USING MATLAB WiTh INTEL CoRE2@2.66GHz.

\section{Simulation Results}

In this section, simulation results for the proposed LPOFDM schemes applied to multicast systems are presented and the performances of the different algorithms are compared with the conventional multicast approach LCG. The generated signal is composed of $N=1024$ subcarriers transmitted in the band $[0.3 ; 20]$ MHz. Perfect synchronization and channel estimation are assumed. A high background noise level of $-110 \mathrm{dBm} / \mathrm{Hz}$ is assumed and the signal is transmitted with respect to a flat PSD of $-50 \mathrm{dBm} / \mathrm{Hz}$ for all users. Results are given for a fixed target symbol error rate (SER) of $10^{-6}$. A constant coding gain $\gamma_{c}$ of $6 \mathrm{~dB}$ for all modulation orders is used and a system margin $\gamma_{m}$ is set to $3 \mathrm{~dB}$. In order to take into account the coding gain and the system margin, the SNR gap $\Gamma$ is given by [8]:

$$
\Gamma(\mathrm{dB})=9.8-\gamma_{c}+\gamma_{m} .
$$


The maximum number of bits per symbol is limited to 10 . The multipath channel model for PLC as proposed in [9] is used. This channel model is randomized to generate enough channels for simulations in multiuser context. The reference model of this channel model is $110 \mathrm{~m}$ link 15-path model which frequency response is given by

$$
H(f)=\sum_{i=1}^{15} g_{i} \cdot e^{\left(a_{0}+a_{1} f^{k}\right)^{d_{i}}} \cdot e^{-2 j \pi f\left(\tau_{i}\right)} .
$$

The parameters of the 15-path model are detailed in [9] and Fig. 4 shows 3 examples of used channel transfer functions.

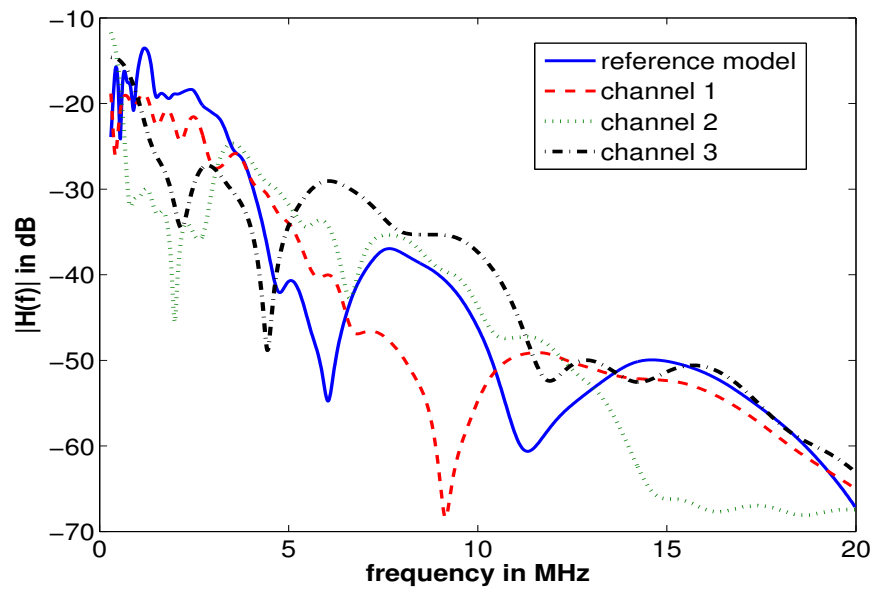

Fig. 4. 15-path reference channel model and generated channels for PLC

Fig. 5 depicts the comparison of proposed LP-OFDM based solutions with conventional LCG method. It shows that the conventional multicast (LCG) bit rate significantly decreases when the number of users increases. The figure also shows that the multicast bit rate is increased by using the linear precoding component. When there is a user with very bad channel conditions, the multicast bit rate significantly decreases. For example, for a 9-user system, about 2 bit per subcarrier are lost compared to a 8-user system. As previously stated, the calculation of the equivalent multicast channel makes the multicast resource allocation equivalent to single user one. Therefore, the difference performance between LCG and LPLCG methods confirms the fact that LP-OFDM offers more bit rate in single user context. The heuristic solution offers better bit rate when the number of users is less than 25 . Thus, the optimization of blocks for each user, before selecting the best case (i.e. heuristic solution), brings better results for lower number of users, but becomes less efficient for higher number of users. This figure confirms also the fact that the improved-LP-LCG method guarantees a better multicast bit rate compared to others for higher number of users and gives performance near the heuristic solution for lower number of users. Compared to the conventional multicast LCG method, the improved-LP-LCG method offers a bit rate gain near $37 \%$ for a 100-user system. This method is quite simpler and can be

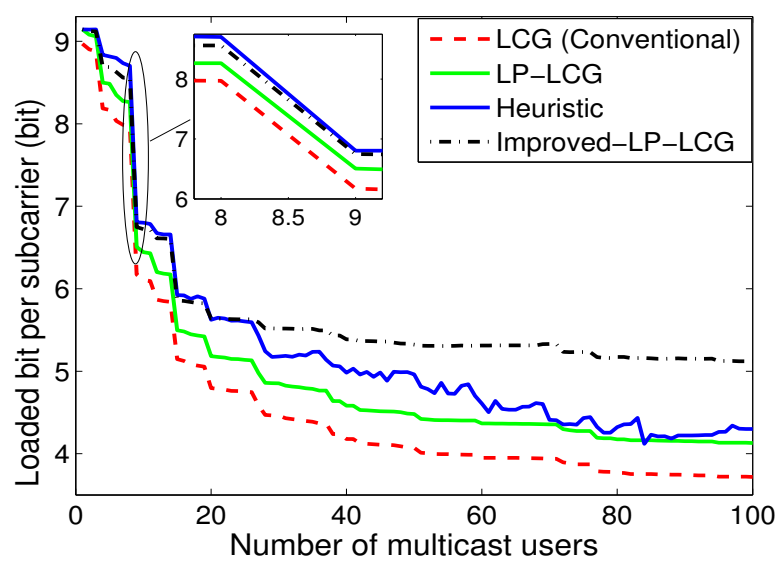

Fig. 5. Comparison of different proposed algorithms

considered for resource allocation solution in multicast OFDM systems for PLC.

\section{CONCLUSION}

In this paper, linear precoding technique has been adapted to multicast OFDM systems in PLC context. To increase the multicast bit rate, different new resource allocation algorithms have been proposed based on linear precoding technique. It has been shown through simulations that proposed algorithms bring a bit rate gain up to $37 \%$ compared to conventional multicast resource allocation method.

\section{ACKNOWLEDGMENT}

The research leading to these results has received funding from the European Community's Seventh Framework Programme FP7/2007-2013 under grant agreement $n^{\circ} 213311$ also referred to as OMEGA.

\section{REFERENCES}

[1] C. Suh, J. Mo, "Resource allocation for multicast services in multicarrier wireless communications," in Proc. of IEEE Infocom 2006, Apr. 2006, Barcelona, Spain.

[2] C. Suh, C. S. Hwang, "Dynamic subchannel and bit allocation for multicast OFDM systems," in Proc. of IEEE PIRMC'04, Sept 2004, Barcelona, Spain.

[3] C.-S. Hwang and Y. Kim, "An adaptive modulation method for multicast communications of hierarchical data in wireless networks," Proc. of IEEE ICC., pp. 896-900, 2002.

[4] A. Maiga, J-Y. Baudais, and J-F. Hélard, "Very High Bit Rate Power Line Communications For Home Networks," IEEE ISPLC09, April 2009, Dresden, Germany.

[5] M. Crussière, J-Y. Baudais, and J-F. Hélard, "Adaptive linear precoded DMT as an efficient resource allocation scheme for power-line communications," Proc. of IEEE GLOBECOM'06, San Francisco, USA, November 2006.

[6] M. Crussière, J-Y. Baudais, and J-F. Hélard, "Adaptive Spread-Sprectrum Multicarrier Multiple-Access Over Wirelines," IEEE JSAC, Vol. 24, No. 7, July 2006.

[7] S. Karabati, P. Kouvelis, and G. Yu, "A min-max-sum resource allocation problem and its applications," Operations Research, 2001, Nov-Dec, Vol. 49, Issue. 6 , 913-922

[8] J. M. Cioffi, "A multicarrier primer," November 1991, ANSI Contribution T1E1.4/91-157, Clearfield, Fla, USA.

[9] M. Zimmermann and K. Dostert,"A multipath model for the powerline channel,” IEEE Trans. Commun., vol. 50, no. 4, pp. 553, Apr. 2002. 